\title{
PD-I Expression in Pre-Treatment Follicular Lymphoma Predicts the Risk of Subsequent High-Grade Transformation
}

This article was published in the following Dove Press journal: OncoTargets and Therapy

\author{
Marie Beck Enemark ${ }^{1,2, *}$ \\ Ida Monrad',* \\ Charlotte Madsen' \\ Kristina Lystlund Lauridsen ${ }^{3}$ \\ Bent Honoré (iD ${ }^{4}$ \\ Trine Lindhardt Plesner ${ }^{5}$ \\ Stephen Jacques Hamilton- \\ Dutoit $\mathbb{D D}^{3}$ \\ Francesco d'Amore' \\ Maja Ludvigsen (iD) ${ }^{1,2}$ \\ 'Department of Hematology, Aarhus \\ University Hospital, Aarhus, Denmark; \\ ${ }^{2}$ Department of Clinical Medicine, \\ Aarhus University, Aarhus, Denmark; \\ ${ }^{3}$ Department of Pathology, Aarhus \\ University Hospital, Aarhus, Denmark; \\ ${ }^{4}$ Department of Biomedicine, Aarhus \\ University, Aarhus, Denmark; \\ ${ }^{5}$ Department of Pathology, Copenhagen \\ University Hospital, Copenhagen, \\ Denmark \\ *These authors contributed equally to \\ this work
}

\begin{abstract}
Purpose: Follicular lymphoma (FL) is an indolent, yet generally incurable neoplasia with a median survival exceeding 10 years. However, a subset of FL patients experiences histological transformation (HT) to a more aggressive lymphoma, in the majority of cases to diffuse large B-cell lymphoma (DLBCL). This affects both the clinical course and the prognostic outcome, resulting in a markedly reduced survival after transformation. Thus, early risk stratification and prediction of patients at risk of HT would be highly valuable in the clinical setting. Here, we investigated the potential of the immune inhibitory programmed death 1 (PD-1) receptor as a biomarker predictive of HT.
\end{abstract}

Patients and Methods: Immunohistochemical staining and quantification by digital image analysis of PD-1 was performed on diagnostic tumor-tissue samples from FL patients with and without subsequent transformation ( $n=34$ and $n=46$, respectively), and on paired samples from the transformed lymphoma $(n=34)$.

Results: At the time of initial FL diagnosis, samples from patients with subsequent HT had significantly higher tumor-tissue expression of PD-1 compared with diagnostic FL samples from patients without subsequent HT $(\mathrm{p}=0.010)$. At the time of transformation, PD-1 expression was significantly reduced $(\mathrm{p}<0.001)$. No difference was observed in intrafollicular PD-1 expression at FL diagnosis between samples from patients with or without HT; however, high intra-follicular levels of PD-1 were associated with significantly shorter transformation-free survival times $(\mathrm{p}<0.043)$.

Conclusion: Our data suggest that pre-treatment tumor-tissue PD-1 expression already predicts the risk of subsequent transformation to DLBCL, as early as the time of FL diagnosis.

Keywords: programmed death 1; PD-1, follicular lymphoma; FL, histological transformation; HT

\section{Introduction}

Follicular lymphoma (FL) is an indolent, yet mostly incurable lymphoproliferative neoplasia derived from germinal center B cells. It is the second most common lymphoid malignancy in the USA and western Europe, accounting for $20 \%$ of all Non-Hodgkin's lymphomas, with a median age at diagnosis of approximately 60-65 years. ${ }^{1-4}$ The disease is generally characterized by an indolent clinical course with good responses to treatment but also recurrent relapses. ${ }^{2}$ The introduction of treatment with CD20 targeting antibodies has further improved the prognosis, resulting in a median survival exceeding 10 years. ${ }^{4-7}$ However, a portion of patients
Correspondence: Maja Ludvigsen Department of Hematology, Aarhus University Hospital, Palle Juul-Jensens Boulevard 99, CII5, Aarhus N 8200, Denmark

Tel +4522859523

Email majlud@rm.dk 
experiences early disease progression, progressive loss of treatment sensitivity and treatment refractoriness. Moreover, a subset of patients experiences histological transformation (HT) to a more aggressive lymphoma, typically diffuse large B-cell lymphoma (DLBCL) or FL grade $3 \mathrm{~B}$. $^{7} \mathrm{HT}$ has a clear adverse impact on the patient's prognosis, with a drastic deterioration in the clinical course and a marked reduction in the median survival after transformation. ${ }^{5,7-10}$

Biomarkers that could reliably predict HT, and thus identify patients at increased risk of transformation, would be highly valuable in the clinical setting. To date, both clinical features and biological markers have been investigated as possible risk factors for HT, ${ }^{7,8,11,12}$ but no definitive biomarkers of transformation have yet been identified. Known factors associated with poor prognosis in FL include advanced Ann Arbor stage, elevated lactate dehydrogenase (LDH), B-symptoms as well as a high Follicular Lymphoma International Prognostic Index (FLIPI) score. ${ }^{5,7,8}$ Our group have previously investigated potential biomarkers predictive of HT. We reported differences in expression of various proteins, including vimentin, GAPDH and aldolase $A$, in diagnostic FL samples from patients with subsequent transformation compared with those without. ${ }^{11-13}$

The importance of the tumor microenvironment in the development, progression, and transformation of FL has been suggested. In fact, multiple studies have identified molecular markers of the non-malignant tumor microenvironment including those associated with the regulation of tumor-infiltrating immune cells, as being correlated to the risk of HT. ${ }^{14-16}$ Among these are immune checkpoint molecules, a group of co-inhibitory receptors that inhibit T-cell effector function upon activation. One such checkpoint protein is the inhibitory programmed death 1 (PD-1) receptor, which is expressed on a variety of hematopoietic cells, especially T cells. ${ }^{17}$ Signaling through PD-1 by its two ligands programmed death ligand 1 and 2 (PD-L1/2) ultimately leads to inhibition of downstream $T$ cell receptor signaling, thereby decreasing cytokine production, effector $\mathrm{T}$ cell proliferation and survival, thus resulting in the state of $\mathrm{T}$ cell exhaustion. $^{17-19}$ PD-1/PD-L1/2 signaling represents a crucial mechanism of immune tolerance, and it serves as a key mechanism exploited by several types of cancers. Through this mechanism the malignant cells acquire the ability to escape anti-tumor immunity, a feature which eventually results in a survival advantage for these cells. ${ }^{17,18}$

PD-1 has previously been investigated in the context of FL and HT, however with conflicting results regarding tumor-tissue PD-1 expression and its association with disease outcome and risk of transformation. ${ }^{18,20-22}$ Studies of the FL tumor microenvironment have consistently shown abundant PD-1 expression by $\mathrm{T}$ cells, but with complex expression patterns complicating the interpretation. ${ }^{21}$

In this study, we examined the potential for tumor PD-1 expression to predict HT in patients with FL. PD-1 expression was evaluated in pretherapeutic diagnostic tumor tissues from FL patients with and without subsequent HT, based on the hypothesis that diagnostic FL samples from patients who subsequently experience HT may show biological characteristics differentiating them from FL without transformation. Furthermore, paired tumor samples from transformed lymphomas at the time of HT were included in the analysis to investigate changes in PD-1 expression from the time of initial FL diagnosis to HT.

\section{Patients and Methods}

\section{Patients}

Analyses were performed on formalin fixed, paraffinembedded (FFPE) tumor-tissue specimens from $80 \mathrm{FL}$ patients, diagnosed at the Department of Hematology, Aarhus University Hospital, Denmark, between 1990 and 2015 with FL grade 1-3A, Figure 1. These included 46 FL patients without HT with at least 10 years of follow up, and 34 patients with a primary FL diagnosis and subsequent histologically confirmed transformation to DLBCL or FL grade $3 \mathrm{~B}$, at least 6 months after the primary $\mathrm{FL}$ diagnosis. For these 34 patients, transformed lymphoma samples from the time of HT were also analyzed. Lymphoma tissues were processed using standard procedures. All biopsies were reviewed by two experienced hematopathologists (TLP and SJHD) and classified according to the 2017 update of the WHO Classification of Tumours of the Haematopoietic and Lymphoid Tissues. ${ }^{1}$ Clinical data on all patients were collected from the Danish Lymphoma Registry (LYFO) ${ }^{23}$ and patient records. These clinical data as well as immunohistochemical data on other putative biological markers in this cohort have previously been published. ${ }^{7,11,12}$ The study is approved by the Danish National Committee on Health Research Ethics (1-10-72-276-13) and the Danish Data Protection Agency (1-16-02-407-13). The requirement for study-specific informed consent was waived due to the retrospective nature of the study and the long follow-up time since inclusion. The study was conducted in accordance with the Declaration of Helsinki. 


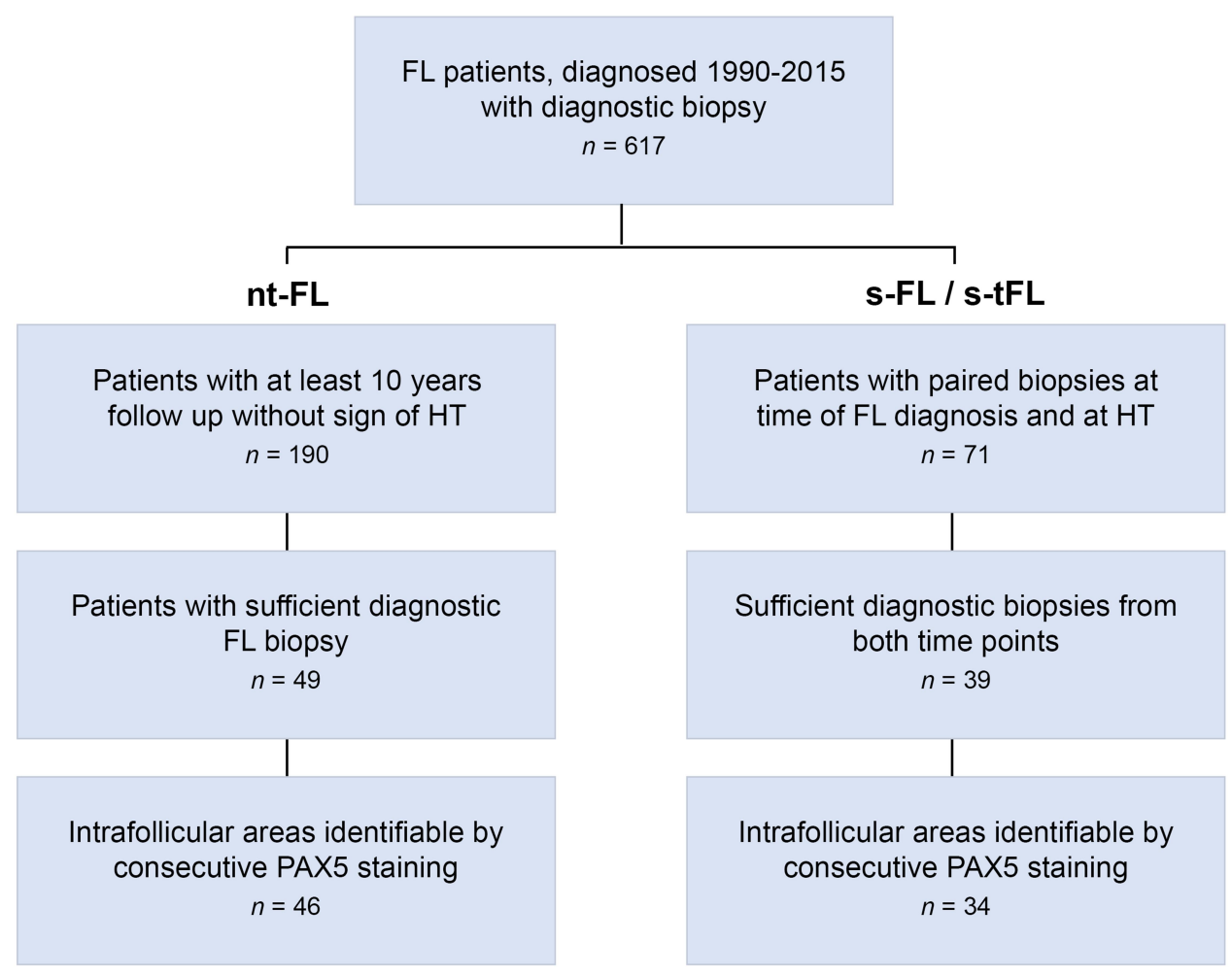

Figure I Consort diagram of the cohort. Pathology reports of all diagnostic biopsies for patients diagnosed with FL at the Department of Hematology, Aarhus University Hospital, Denmark between 1990-2015 were reviewed. Patients were categorized as non-transforming FL (nt-FL) if they showed no sign of HT in a follow-up period of at least 10 years. Patients were categorized as subsequently transforming FL (s-FL) if the patient presented with a primary diagnosis of FL grade I-IIIA (s-FL) and subsequently, at least 6 months later, with a biopsy of histologically transformed FL (s-tFL). Patients were included when sufficient FFPE tumor tissue were available from an excision or large needle biopsy. Only s-FL/s-tFL patients with a complete pair of biopsies from time of initial FL diagnosis and time of HTwere included in the transformed sub-cohort. If necessary, to complete a pair, archived FFPE tissue were collected from other lymphoma treating centers in the central and southern region of Jutland, Denmark. All biopsies were reviewed and classified according to the 2017 update of the WHO Classification of Tumours of the Haematopoietic and Lymphoid Tissues. ' Furthermore, samples were included if a consecutive parallel tissue section stained with PAX5 were able to identify B cell areas, and thus intrafollicular areas, within the biopsy section. In total, we included samples from 46 nt-FL patients with no subsequent transformation, and 34 patients with s-FL/s-tFL biopsy pairs.

Abbreviations: FFPE, formalin-fixed paraffin embedded; FL, follicular lymphoma; HT, histological transformation; nt-FL, non-transforming FL, s-FL, subsequently transforming FL; s-tFL, histologically transformed lymphoma.

\section{Immunohistochemical Staining for PD-I}

Four- $\mu \mathrm{m}$ FFPE tissue sections were immunohistochemically stained on the Ventana BenchMark Ultra automated slide stainer (Ventana Medical Systems, Roche, Oro Valley, AZ) using standard methods. Primary monoclonal mouse anti-human anti-PD-1 (Product no. 315M-96, NAT105, Cell Marque, CA, USA) was used at a dilution of 1:100. Primary antibody detection was performed using the Ventana OptiView DAB detection kit (Ventana Medical Systems, Roche). Sections of appendix, tonsil, liver and pancreas were included on each slide as positive/negative controls. ${ }^{24}$

\section{Digital Image Analysis}

Stained slides were scanned at a magnification of x20 using the Hamamatsu Nanozoomer 2.0HT scanner (Hamamatsu, Shizuoka, Japan). Staining quantification was performed using Visiopharm Integrator system
2018.09 (Visiopharm A/S, Hoersholm, Denmark). As previously described, ${ }^{12}$ regions of interest (ROI) on whole biopsy sections were manually outlined, defining the area of tissue suitable for staining quantification, excluding distinct areas of non-lymphoid tissue, fatty tissue, tissue artefacts etc. An analysis protocol package (APP) was designed by manual image class designation to appropriate tissue areas (tissue background, weak-, intermediate- and strong staining intensity) followed by APP training based on tissue sections representing differential expression levels of the biomarker. Area fractions (AFs) were used as staining quantification outputs and were defined as the stained area normalized to the area of the ROI. ${ }^{12}$ PD-1 expression levels were based on AFs of strong intensity staining. Intrafollicular regions were manually outlined guided by a consecutive parallel section, stained with PAX5 to identify B cell areas in the biopsy. ${ }^{11}$ 


\section{Statistical Analysis}

Differences in AFs of FL diagnostic samples from patients with or without subsequent HT was assessed using Student's $t$-test and paired $t$-test. Differences in clinicopathological features were assessed using Fisher's exact test, and correlations of biomarker expression to clinicopathological features were evaluated using Spearman rank test. Time-related endpoints were analyzed using the Kaplan Meier method, with transformation-free survival (TFS) as endpoint. TFS was defined as the time from initial FL diagnosis to the date of histologically confirmed HT or censoring. ${ }^{12}$ Determination of the optimal cutoff value for high versus low expression level of PD-1 on tumor sections for TFS analysis was established by a ROC analysis with the optimal cutoff point calculated using Youden's index. This cutoff value corresponded to the 75th percentile of PD-1 expression and therefore, the cutoff values for TFS are denoted as the 75th percentile throughout the manuscript. Binary logistic analysis was used to calculate odds ratios (OR).

$\mathrm{P}$-values below 0.05 were considered statistically significant. Statistical analyses were performed in STATA version 15.1 (StataCorp).

\section{Results}

The patient cohort comprised a total of 80 FL patients, including 41 males and 39 females, Table 1 . The age at diagnosis ranged from 25 to 83 years with a median age at diagnosis of 55 years. FL patients with subsequent transformation $(n=34)$ had a higher risk profile compared with patients without HT $(n=46)$, with a more advanced Ann Arbor stage and higher FLIPI score.

Immunohistochemical evaluation of PD-1 revealed membranous staining of specific cellular subsets within the lymphoma samples in both non-follicular and intrafollicular areas, with positively stained areas predominantly being located within lymphoid tissue, Figure 2A and $\mathrm{B}$. At the time of initial FL diagnosis, samples from patients with subsequent HT had significantly higher expression of PD-1 (95\% confidence interval (CI): $\mathrm{AF}=1.99 \cdot 10^{-3}-4.79 \cdot 10^{-3}$ ) compared with samples from patients without subsequent transformation $(95 \% \mathrm{CI}$ : $\left.\mathrm{AF}=3.92 \cdot 10^{-4}-2.91 \cdot 10^{-3}, \mathrm{p}=0.010\right)$, Figure $2 \mathrm{C}$. Lymphoma samples from the time of HT had a significantly lower expression of PD-1 compared with their paired diagnostic FL samples $(95 \%$ CI: $\left.\mathrm{AF}=2.69 \cdot 10^{-5}-7.57 \cdot 10^{-4}, \mathrm{p}<0.001\right)$, Figure 2C. No significant difference was found when analyzing PD-1 expression exclusively localized within intra-follicular areas, Figure 2D. There was no correlation between PD1 expression levels and any of the clinicopathological features.

High levels of strong intensity PD-1 expression at the time of primary FL diagnosis were not found to be associated with a significantly shorter TFS $(p=0.200)$ when assessing the ROI of the whole tumor tissue section; however, OR showed that the odds of tumors with high intratumoral PD-1 expression subsequently transforming were higher than those with low intratumoral PD-1 expression (OR: 4.67, 95\% CI: 1.56-13.97), Figure 2E. When analyzing exclusively intra-follicular PD-1 expression, TFS was significantly shorter among patients with high PD-1 expression level ( $<<0.043$, OR: 9.33, 95\% CI: 2.74-31.83), Figure 2F.

\section{Discussion}

In this study, we used digital computer-assisted analysis to help quantify immunohistochemical PD-1 expression, and to assess the potential of this biomarker to predict HT in FL. We found that strong intensity PD-1 expression was significantly higher in FL diagnostic samples from patients who subsequently experienced transformation to a highgrade lymphoma, compared with those who did not. Additionally, PD-1 expression was significantly lower in lymphoma samples after HT. This decrease in PD-1 expression after HT may well reflect the reduced levels of immune cell infiltrates, characteristic of DLBCL., ${ }^{4,25}$ When analyzing exclusively in intra-follicular areas, no differences in PD-1 expression were found in FL diagnostic samples.

With a cutoff at the 75th percentile, differences in high versus low PD-1 expression correlated with a slight trend towards shorter TFS among patients with high PD-1 expression, although these were not significantly different. However, when focusing on PD-1 expression exclusively within follicles, TFS were significantly shorter among patients with high PD-1 expression levels.

Results similar have previously been reported by Blaker et al who found high intra-follicular PD-1 levels in FL to be associated with subsequent transformation compared with those cases without $(n=52) .{ }^{15}$ On the contrary, Smeltzer et al $(n=58)$ and Wahlin et al $(n=70)$ found high numbers of intra-follicular PD- $1^{+} \mathrm{T}$ cells in FL to be an indicator of good disease outcome, and Smeltzer et al further reported shorter time to transformation. ${ }^{16,26}$ In 
Table I Patients' Clinico-Pathological Features

\begin{tabular}{|c|c|c|c|c|}
\hline Characteristics & $\begin{array}{l}\text { All } n=80 \\
n(\%)\end{array}$ & $\begin{array}{l}\text { nt-FL n=46 } \\
n(\%)\end{array}$ & $\begin{array}{l}s-F L ~ n=34 \\
n(\%)\end{array}$ & P-value \\
\hline \multicolumn{5}{|l|}{ Sex } \\
\hline Male & $4 \mid(5 I)$ & $20(43)$ & $21(62)$ & NS \\
\hline Female & $39(49)$ & $26(57)$ & $13(38)$ & \\
\hline \multicolumn{5}{|l|}{ Age at FL diagnosis } \\
\hline Median & 55 & 55 & 56 & NS \\
\hline Range & $25-83$ & $35-83$ & $25-78$ & \\
\hline \multicolumn{5}{|l|}{ Ann Arbor stage } \\
\hline $\mathrm{I}-\mathrm{II}$ & $27(34)$ & $23(50)$ & $4(12)$ & $<0.001$ \\
\hline III-IV & $51(64)$ & $22(48)$ & $29(85)$ & \\
\hline Unknown & $2(2)$ & I (2) & I (3) & \\
\hline \multicolumn{5}{|l|}{ FLIPI } \\
\hline Low & $30(37)$ & $24(52)$ & $6(18)$ & 0.002 \\
\hline Intermediate & $27(34)$ & $15(33)$ & $12(35)$ & \\
\hline High & $19(24)$ & $5(11)$ & $14(4 \mid)$ & \\
\hline Unknown & $4(5)$ & $2(4)$ & $2(6)$ & \\
\hline \multicolumn{5}{|l|}{ LDH-elevation } \\
\hline Yes & II (I4) & $3(7)$ & $8(23)$ & NS \\
\hline No & $65(8 \mathrm{I})$ & $4 \mid(89)$ & $24(7 \mathrm{I})$ & \\
\hline Unknown & $4(5)$ & $2(4)$ & $2(6)$ & \\
\hline \multicolumn{5}{|l|}{ B-symptoms } \\
\hline Yes & $21(26)$ & $9(20)$ & $12(35)$ & NS \\
\hline No & $56(70)$ & $36(78)$ & $20(59)$ & \\
\hline Unknown & $3(4)$ & I (2) & $2(6)$ & \\
\hline \multicolumn{5}{|l|}{ Performance score } \\
\hline$<2$ & $75(94)$ & $43(94)$ & $32(94)$ & NS \\
\hline$\geq 2$ & $2(2)$ & $2(4)$ & $0(0)$ & \\
\hline Unknown & $3(4)$ & I (2) & $2(6)$ & \\
\hline \multicolumn{5}{|l|}{ Bone marrow } \\
\hline Involvement & $22(27)$ & $8(17)$ & $14(4 \mid)$ & NS \\
\hline No & 47 (59) & $31(68)$ & $16(47)$ & \\
\hline Unknown & II (I4) & $7(15)$ & $4(12)$ & \\
\hline \multicolumn{5}{|l|}{ Anemia } \\
\hline Yes & $5(6)$ & I (2) & $4(12)$ & NS \\
\hline No & $72(90)$ & $44(96)$ & $28(82)$ & \\
\hline Unknown & $3(4)$ & I (2) & $2(6)$ & \\
\hline \multicolumn{5}{|l|}{ FL histology } \\
\hline FL NOS & $0(0)$ & $0(0)$ & $0(0)$ & NS \\
\hline FL grade $1-2$ & $66(83)$ & $36(78)$ & $30(88)$ & \\
\hline FL grade $3 \mathrm{~A}$ & $14(17)$ & $10(22)$ & $5(12)$ & \\
\hline \multicolumn{5}{|l|}{ Initial treatment } \\
\hline Alkylator-based & $23(29)$ & $15(33)$ & $8(24)$ & NA \\
\hline Antracyclin-based & $6(8)$ & $2(4)$ & $4(12)$ & \\
\hline Chlorambucil & $18(23)$ & II (24) & $7(21)$ & \\
\hline Rituximab only & $8(10)$ & $2(4)$ & $6(18)$ & \\
\hline Radiation only & $7(9)$ & $5(11)$ & $2(6)$ & \\
\hline Watch and wait & $13(16)$ & $8(17)$ & $5(15)$ & \\
\hline Other & $5(2)$ & $3(7)$ & $2(6)$ & \\
\hline R-Chemo & $24(30)$ & $10(22)$ & $14(4 \mid)$ & \\
\hline
\end{tabular}

(Continued)
Table I (Continued).

\begin{tabular}{|c|c|c|c|c|}
\hline Characteristics & $\begin{array}{l}\text { All } n=80 \\
n(\%)\end{array}$ & $\begin{array}{l}n t-F L n=46 \\
n(\%)\end{array}$ & $\begin{array}{l}s-F L n=34 \\
n(\%)\end{array}$ & P-value \\
\hline \multicolumn{5}{|l|}{$\begin{array}{l}\text { PD-I expression, } \\
\text { whole biopsy }\end{array}$} \\
\hline Low & $60(75)$ & $40(87)$ & 20 (59) & 0.008 \\
\hline High & $20(25)$ & $6(13)$ & $14(4 \mid)$ & \\
\hline \multicolumn{5}{|l|}{$\begin{array}{l}\text { PD-I expression, } \\
\text { follicles }\end{array}$} \\
\hline Low & $60(75)$ & $42(91)$ & $18(53)$ & $<0.001$ \\
\hline High & $20(25)$ & 4 (9) & $16(47)$ & \\
\hline
\end{tabular}

Notes: PD-I analysis was performed with the cutoff for high versus low PD-I expression level based on the 75 percentile of expression $(A F=0.003)$.

Abbreviations: FL, follicular lymphoma; FLIPI, follicular lymphoma international prognostic index; FL NOS, follicular lymphoma not otherwise specified; LDH, lactate dehydrogenase; NA, not applicable; NS, not significant; nt-FL, nontransforming FL; PD-I, programmed death I; s-FL, subsequently transforming FL.

contrast, Szumera-Ciećkiewicz et al found interfollicular PD-1 expression predictive of poorer patient prognosis $(n=71) .^{22}$ Thus, contradictory results have previously been reported regarding the predictive value of PD-1 in FL.

Comparison of results between groups poses a challenge, as the studies have used different methodological approaches. Thus, Blaker et al, Wahlin et al and Szumera-Ciećkiewicz et al used tissue microarrays (which focus on neoplastic tissue and may not always be representative of the whole tumor), while in the present study we used whole tissue sections. ${ }^{15,22,26}$ Moreover, the studies show differences in which tissue compartments were included in the analyses, ie, whole biopsy versus non-follicular versus intra-follicular. In spite of the inconsistencies in results reported by the different studies, we believe that the possible part played by PD-1 in the process of HT in FL should not be overlooked. However, clearer understanding of the role of PD-1 as a biomarker in FL will depend on further investigation.

There is a solid rationale for analyzing FL tumor tissues at a compartmental niveau, subdivided into intra-follicular and non-follicular areas, since these regions harbor different populations of $\mathrm{T}$ cell subsets which could possibly affect malignant growth in different ways. When defining the intra-follicular areas, there is a risk of introducing subjective bias. However, follicles were outlined guided by a parallel PAX5-stained section to show the location of B cells in the same biopsy and reviewed by an experienced hematopathologist.

FL has been characterized by the presence of significant numbers of $\mathrm{T}$ cell subsets in the tumor microenvironment. ${ }^{27}$ 

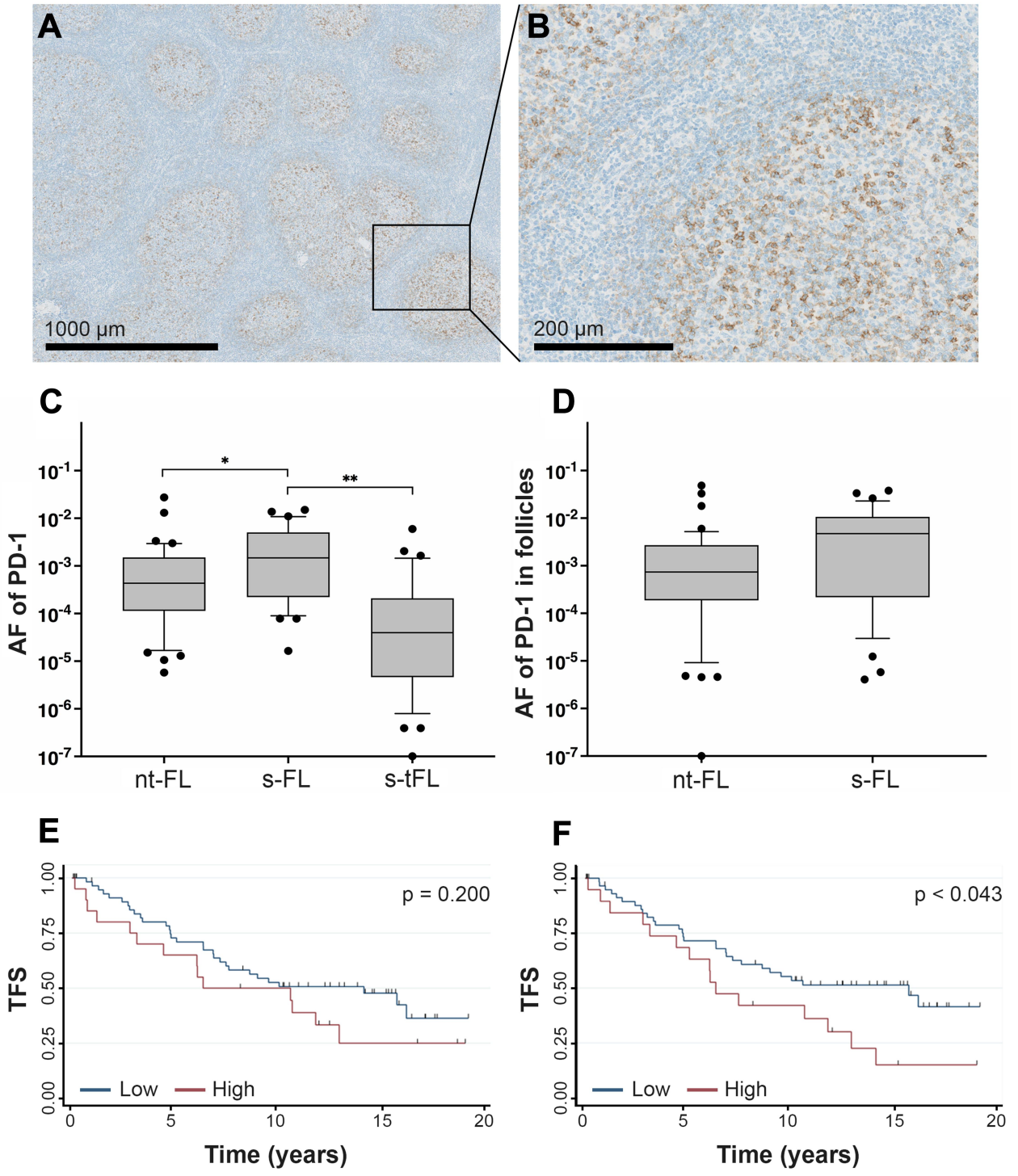

Figure 2 Expression of PD-I and outcome analysis. (A and B) Representative images of tumor tissue stained for PD-I. (C) Area fractions of strong intensity PD-I staining in FL diagnostic samples from patients with subsequent transformation and patients without, and in lymphoma samples from time of $H T$ diagnosis; ${ }^{*} \mathrm{P}<0.0 \mathrm{I}$, ** $\mathrm{p}<0.00 \mathrm{I}$. (D) Area fractions of PD-I staining exclusively localized in intra-follicular areas. (E) Association between PD-I expression in whole biopsies and TFS (F) Association between exclusively intra-follicular PD-I expression and TFS.

Abbreviations: FL, follicular lymphoma; HT, histological transformation; nt-FL, non-transforming FL; PD-I, programmed death I; s-FL, subsequently transforming FL; s-tFL, histologically transformed lymphoma; TFS, transformation-free survival.

$\mathrm{T}$ cells located in follicles are most likely to be of the $\mathrm{T}$ follicular helper subtype, which are known to promote $\mathrm{B}$ cell growth and lymphomagenesis in FL., ${ }^{4,5}$ Wahlin et al suggested that expression of inhibitory receptors on these cells could arguably dampen their stimulatory effects, causing improvements in the prognosis of FL and possibly lowering the risk of transformation. ${ }^{26}$ However, as the results found in both the present study and those reported by Blaker et al are conflicting, more evidence is needed to fully establish the role of PD-1 in FL. ${ }^{15}$

In addition to inhibitory receptors, Yang et al found costimulatory receptors CD27 and CD28 to be frequently lost on T cell subsets in FL. ${ }^{28}$ These findings of increased inhibitory signals and decreased stimulatory signals supports previous studies describing a suppressive tumor microenvironment in $\mathrm{FL}^{28}$ Furthermore, Yang et al found that overall PD-1 expression were not significantly associated with patient outcome. However, when separating T cells into different PD-1 positive subsets based on their phenotype, differences in patient outcome were seen depending on the subset. ${ }^{28}$

In this study, we analyzed whole tissue sections, thus allowing changes in the tumor microenvironment to be included. The association between high PD-1 levels and 
shorter time to transformation found by us could be interpreted as the malignant cells exploiting the PD-1/PD-L1/2 pathway, thereby leading to immune exhaustion and impaired anti-tumor immunity.

Underlying mechanisms driving HT remains a subject of investigation with different proposed pathways leading to the transformation event. One hypothesis assumes a continuous accumulation of genetic alterations within the original FL cell population, while other hypothesizes that transformation occurs via expansion of a small subset of transformed FL subclones becoming dominating at HT. ${ }^{29-31}$ Previous studies have suggested possible affected pathogenic mechanisms to be involved in transformation of FL in regard to impairment of DNA damage response, oxidative stress, immune regulation, and chronic inflammation, which all have previously been described to be involved in malignant development. ${ }^{29,31-33}$ Hypothetically, upregulated expression of PD-1 could very well be caused by impaired immune regulation and chronic inflammation, thereby exhausting the immune system and eventually leading to HT. However, larger in-depth mechanistic studies are needed to ascertain this hypothesis.

Prior implementation of this putative biomarker predictive of transformation in a clinical setting, validation of our findings in larger and independent cohorts is a prerequisite for prospective use in risk assessment of FL patients. In the present study, the sample size was limited as only patients with sufficient FFPE tumor biopsies available at both FL diagnosis and at HT for patients with subsequent transformation and non-transforming FL with at least 10 years follow-up were included. This resulted in $34 \mathrm{FL}$ patients with subsequent transformation (and paired samples) and 46 patients without transformation. Thus, inclusion based on availability introduced that the patients with subsequent transformation presented with advanced risk profiles in regard to increased Ann Arbor stage and FLIPI score. This could potentially bias the analyses, however, we found that tumor PD-1 expression did not correlate with any clinicopathological features, neither Ann Arbor stage nor FLIPI score. Thus, this implies that presumably an independent impact of PD-1 expression is not correlated with the adverse risk profile in the subsequently transforming FL group.

The results presented support an important role for PD1 in the eventual progression of FL, although validation of our findings will be necessary before PD-1 expression analysis can be recommended for use as a relevant prognostic or predictive biomarker in the clinical routine.

\section{Conclusion}

This study demonstrates the value of PD-1 expression for predicting transformation in FL. We found high PD-1 expression at FL diagnosis predictive of the risk of later histological transformation, and high intra-follicular expression of PD-1 to be associated with shorter time to transformation. More data are needed to provide clear insights into the function of PD-1 in the process of histological transformation in FL tumors and fully elucidate its potential as a predictive biomarker of HT.

\section{Abbreviations}

$\mathrm{AF}$, area fraction; APP, application protocol package; CI, confidence interval; DLBCL, diffuse large B-cell lymphoma; FFPE, formalin-fixed paraffin embedded; FL, follicular lymphoma; FLIPI, follicular lymphoma international prognostic index; HT, histological transformation; LDH, lactate dehydrogenase; LYFO, the Danish lymphoma registry; PD-1, programmed death 1 ; PD-L1/2, programmed death ligand $1 / 2$; TFS, transformation-free survival; TMA, tissue microarray; TME, tumor microenvironment; ROI, region of interest.

\section{Ethics Approval}

The study is approved by the Danish National Committee on Health Research Ethics (1-10-72-276-13) and the Danish Data Protection Agency (1-16-02-407-13). The requirement for study-specific informed consent was waived due to the retrospective nature of the study and the long follow-up time since inclusion.

\section{Acknowledgments}

This study was funded by Department of Clinical Medicine, Aarhus University, Merchant Einar Willumsen's Memorial Foundation, the Danish Lymphoma Group, a donation from Peter and Alice Madsen, Knud and Edith Eriksen's Memorial Foundation, Eva and Henry Frænkel's Memorial Foundation, and the Central Denmark Region.

\section{Author Contributions}

All authors made a significant contribution to the work reported. MBE, IM, TLP, CM and ML conceptualized the study and all authors agreed on the study design. MBE, IM, CM, KLL, BH, FdA, SJHD, and ML executed the experiments and the statistical analyses. TLP and SJHD revised the pathological diagnoses. $\mathrm{CM}$ and FdA did 
clinical data acquisition and build the patient cohort. All authors contributed to data interpretation. MBE, IM, and ML wrote the initial manuscript draft. All authors critically reviewed the manuscript and approved the final version. All authors agreed on the journal to which the article is submitted and all authors agreed to take responsibility and be accountable for the contents of the article.

\section{Disclosure}

All authors declare no other relevant conflicts of interest in this work. Prof. Dr. Bent Honoré received funding from Bayer for studying the effect of aflibercept on experimental branch retinal vein occlusion. He holds shares from Novo Nordisk A/S and Genmab A/S. In addition, Prof. Dr. Bent Honoré has a patent Biomarkers for Pulmonary Embolism in Exhaled Breath Condensate pending. Bayer, Novo Nordisk $\mathrm{A} / \mathrm{S}$, and Genmab A/S had no influence on the study design, analyses and reporting of the results.

\section{References}

1. Swerdlow SH, Campo E, Pileri SA, et al. The 2016 revision of the World Health Organization classification of lymphoid neoplasms. Blood. 2016;127(20):2375-2390. doi:10.1182/blood-2016-01-643569

2. Freedman A, Jacobsen E. Follicular lymphoma: 2020 update on diagnosis and management. Am J Hematol. 2020;95(3):316-327. doi:10.1002/ajh.25696

3. Huet S, Sujobert P, Salles G. From genetics to the clinic: a translational perspective on follicular lymphoma. Nat Rev Cancer. 2018;18(4):224-239. doi:10.1038/nrc.2017.127

4. Lackraj T, Goswami R, Kridel R. Pathogenesis of follicular lymphoma. Best Pract Res Clin Haematol. 2018;31(1):2-14. doi:10.1016/j.beha.2017.10.006

5. Kridel R, Sehn LH, Gascoyne RD. Pathogenesis of follicular lymphoma. J Clin Invest. 2012;122(10):3424-3431. doi:10.1172/ JCI63186

6. Madsen C, Clausen MR, Plesner TL, et al. Up-front rituximab maintenance improves outcome in patients with follicular lymphoma: a collaborative Nordic study. Blood Adv. 2018;2(13):1562-1571. doi:10.1182/bloodadvances.2018017673

7. Madsen C, Plesner TL, Bentzen HH, et al. Real world data on histological transformation in patients with follicular lymphoma: incidence, clinico-pathological risk factors and outcome in a nationwide Danish cohort. Leuk Lymphoma. 2020:1-11.

8. Kridel R, Sehn LH, Gascoyne RD. Can histologic transformation of follicular lymphoma be predicted and prevented? Blood. 2017;130 (3):258-266. doi:10.1182/blood-2017-03-691345

9. Montoto S, Fitzgibbon J. Transformation of indolent B-cell lymphomas. J Clin Oncol. 2011;29(14):1827-1834. doi:10.1200/ JCO.2010.32.7577

10. Wagner-Johnston ND, Link BK, Byrtek M, et al. Outcomes of transformed follicular lymphoma in the modern era: a report from the National LymphoCare Study (NLCS). Blood. 2015;126(7):851-857. doi:10.1182/blood-2015-01-621375

11. Madsen C, Lauridsen KL, Plesner TL, et al. High intratumoral expression of vimentin predicts histological transformation in patients with follicular lymphoma. Blood Cancer J. 2019;9(4):35.
12. Monrad I, Madsen C, Lauridsen KL, et al. Glycolytic biomarkers predict transformation in patients with follicular lymphoma. PLoS One. 2020;15(5):e0233449. doi:10.1371/journal.pone.0233449

13. Ludvigsen M, Madsen C, Kamper P, et al. Histologically transformed follicular lymphoma exhibits protein profiles different from both non-transformed follicular and de novo diffuse large B-cell lymphoma. Blood Cancer J. 2015;5(3):e293. doi:10.1038/ bcj. 2015.18

14. Sugimoto T, Watanabe T. Follicular lymphoma: the role of the tumor microenvironment in prognosis. J Clin Exp Hematop. 2016;56 (1):1-19. doi:10.3960/jslrt.56.1

15. Blaker YN, Spetalen S, Brodtkorb M, et al. The tumour microenvironment influences survival and time to transformation in follicular lymphoma in the rituximab era. $B r J$ Haematol. 2016;175 (1):102-114. doi:10.1111/bjh.14201

16. Smeltzer JP, Jones JM, Ziesmer SC, et al. Pattern of CD14+ follicular dendritic cells and PD1+ $\mathrm{T}$ cells independently predicts time to transformation in follicular lymphoma. Clin Cancer Res. 2014;20 (11):2862-2872. doi:10.1158/1078-0432.CCR-13-2367

17. Muenst S, Soysal SD, Tzankov A, Hoeller S. The PD-1/PD-L1 pathway: biological background and clinical relevance of an emerging treatment target in immunotherapy. Expert Opin Ther Targets. 2015;19(2):201-211. doi:10.1517/14728222.2014.980235

18. Xia Y, Jeffrey Medeiros L, Young KH. Signaling pathway and dysregulation of PD1 and its ligands in lymphoid malignancies. Biochim Biophys Acta. 2016;1865(1):58-71. doi:10.1016/j. bbcan.2015.09.002

19. Wherry EJ, Kurachi M. Molecular and cellular insights into T cell exhaustion. Nat Rev Immunol. 2015;15(8):486-499. doi:10.1038/ nri3862

20. Gravelle P, Burroni B, Péricart S, et al. Mechanisms of PD-1/PD-L1 expression and prognostic relevance in non-Hodgkin lymphoma: a summary of immunohistochemical studies. Oncotarget. 2017;8 (27):44960-44975. doi:10.18632/oncotarget.16680

21. Xu-Monette ZY, Zhou J, Young KH. PD-1 expression and clinical PD-1 blockade in B-cell lymphomas. Blood. 2018;131(1):68-83. doi:10.1182/blood-2017-07-740993

22. Szumera-Ciećkiewicz A, Poleszczuk J, Kuczkiewicz-Siemion O, et al. PD1 distribution pattern, regardless of the cell origin, is an independent microenvironmental prognostic factor for progression-free survival in follicular lymphoma. Pathol Res Pract. 2020;216(10):153096. doi:10.1016/j.prp.2020.153096

23. Arboe B, El-Galaly TC, Clausen MR, et al. The Danish national lymphoma registry: coverage and data quality. PLoS One. 2016;11 (6):e0157999. doi:10.1371/journal.pone.0157999

24. Matos LL, Trufelli DC, de Matos MG, da Silva Pinhal MA. Immunohistochemistry as an important tool in biomarkers detection and clinical practice. Biomark Insights. 2010;5:9-20. doi:10.4137/ BMI.S2185

25. Lossos IS, Gascoyne RD. Transformation of follicular lymphoma. Best Pract Res Clin Haematol. 2011;24(2):147-163. doi:10.1016/j. beha.2011.02.006

26. Wahlin BE, Aggarwal M, Montes-Moreno S, et al. A unifying microenvironment model in follicular lymphoma: outcome is predicted by programmed death-1-positive, regulatory, cytotoxic, and helper T cells and macrophages. Clin Cancer Res. 2010;16(2):637-650. doi:10.1158/1078-0432.CCR-09-2487

27. Yang ZZ, Kim HJ, Villasboas JC, et al. Expression of LAG-3 defines exhaustion of intratumoral PD-1(+) T cells and correlates with poor outcome in follicular lymphoma. Oncotarget. 2017;8 (37):61425-61439. doi:10.18632/oncotarget.18251

28. Yang ZZ, Kim HJ, Villasboas JC, et al. Mass cytometry analysis reveals that specific intratumoral CD4(+) T cell subsets correlate with patient survival in follicular lymphoma. Cell Rep. 2019;26(8):21782193.e2173. doi:10.1016/j.celrep.2019.01.085 
29. Mendez M, Torrente M, Provencio M. Follicular lymphomas and their transformation: past and current research. Expert Rev Hematol. 2017;10(6):515-524. doi:10.1080/17474086.2017 .1326812

30. Okosun J, Montoto S, Fitzgibbon J. The routes for transformation of follicular lymphoma. Curr Opin Hematol. 2016;23(4):385-391. doi:10.1097/MOH.0000000000000255

31. Casulo C, Burack WR, Friedberg JW. Transformed follicular non-Hodgkin lymphoma. Blood. 2015;125(1):40-47. doi:10.1182/ blood-2014-04-516815
32. Găman M-A, Epingeac M, Găman A. The evaluation of oxidative stress and high-density lipoprotein cholesterol levels in diffuse large B-cell lymphoma. Revista de Chimie. 2019;70:977-980. doi:10.37358/RC.19.3.7043

33. Gaman AM, Buga AM, Gaman MA, Popa-Wagner A. The role of oxidative stress and the effects of antioxidants on the incidence of infectious complications of chronic lymphocytic leukemia. Oxid Med Cell Longev. 2014;2014:158135. doi:10.1155/2014/158135

\section{Publish your work in this journal}

OncoTargets and Therapy is an international, peer-reviewed, open access journal focusing on the pathological basis of all cancers, potential targets for therapy and treatment protocols employed to improve the management of cancer patients. The journal also focuses on the impact of management programs and new therapeutic

Submit your manuscript here: https://www.dovepress.com/oncotargets-and-therapy-journal agents and protocols on patient perspectives such as quality of life, adherence and satisfaction. The manuscript management system is completely online and includes a very quick and fair peer-review system, which is all easy to use. Visit http://www.dovepress.com/ testimonials.php to read real quotes from published authors. 